\title{
Coma Preceded by Severe Dysphagia in Type II Diabetes Mellitus
}

\author{
Keiji Murakami, MD, Seiki Nambu, MD, Motoo Tsushima, MD, \\ Yasuko Nishioheda, MD, Hideki KoH, MD, Rikurou Hayashi, MD, \\ Hajime ToHo, MD and Masao IKedA, MD
}

\begin{abstract}
A 62-year-old man with 11 years' duration of type II diabetes was hospitalized because of non-ketotic diabetic coma. He had never noted any symptoms of swallowing difficulty until 3 days before admission when they developed gradually and he became comatous. He had never received special medication such as diuretics except anticonvulsants. Even after the recovery from diabetic coma he could hardly swallow solid foods or saliva for about 15 days, but these symptoms subsided gradually. Motility dysfunction of esophagus and pharynx in diabetes mellitus, in most cases they are mild, has been described, although diabetic coma preceded by dysphagia has not been documented except in one report. We studied, therefore, the autonomic nerve function of the present patient and discussed the relationship between dysphagsa and diabetic coma together with the description of the clinical course of this patient. The relatienship between this case and the previously reported cases were compared in terms of the sex, age, txpe of diabetes, clinical course, medication and the automic nerve function.
\end{abstract}

Key Words: Non-ketotic diabetic coma, Esophagus, Motility dysfunction, Autonomic nerve function, Autonomic neuropathy, Dysphagia.

Motility dysfunction of the esophagus and pharynx has been described in diabetes mellitus, but symptoms such as dysphagia, retrosternal discomfort and heartburn are uncommon $^{1-7)}$. These motility changes are presumed to be due to autonomic neuropathy of vagal nerve ${ }^{8)}$, because of the similarlity of motility changes following vagal section and its preponderance in diabetics with severe autonomic neuropathy. Recently, we have experienced a case of nonketotic diabetic coma with severe dysphagia, who had never noted any symptoms of swallowing difficulty until 3 days before admission. Diabetic coma preceded by severe dysphagia has not been known except in one report ${ }^{9)}$. Two such cases in femal type II diabetics were indeed reported, but they were administered diuretics, and their autonomic nerve function was not investigated.

In the present report, we describe the clinical course of the patient together with the results of the autonomic nerve function test. In addition, relationship between dysphagia and diabetic coma is discussed.

\section{METHODS}

All tests for autonomic nerve function based on cardiovascular reflexes were carried out on the same day with 20 minutes' intervals after his recovery for the daily activity.

Blood pressure response to standing:

From Division of Atherosclerosis and Metabolism, Department of Internal Medicine, National

Cardiovascular Genter, Suita

Received for publication February 15, 1982.

Reprint request to: Keiji Murakami, MD, Division of Atherosclerosis and Metabolism Department of Internal Medicine, National Gardiovascular Center, 125, 5 cho-me Fujishirodai, Suita, Osaka, 565, Japan 
After 20 minutes' bed rest, blood pressure change to quick standing was measured by cuff sphygmomanometer. Blood pressure response to handgrip: Using handgrip dynamometer, standardised at $30 \%$ of the maximum voluntary contraction, blood pressure change was measured before and after 5 minutes' sustained handgrip on bed rest ${ }^{10)}$. Heart rate response to standing: This test was also carried out with continous ECG recording in the course of the test for blood pressure response to standing. The length of each R-R interval was measured with ruler and recorded. "30: 15" ratio was calculated from the difference of the R-R intervals at 15 th and 30 th beats after standing $^{11)}$. Beat-to-beat variation during deep breathing: EGG was recorded during a deep respiration with 6 successive breaths on the bed rest ${ }^{12)}$. The individual cycle consisted of 5 seconds' inspiration and 5 second' expiration. R-R intervals during inspiration and expiration were measured with ruler and the difference between the maximum and minimum $\mathrm{R}-\mathrm{R}$ intervals in the individual respiration cycle were calculated. It was expressed at the mean heart rate difference in 6 successive breaths.

\section{CASE REPORT}

A 62-year-old man with type II diabetes of 11 years' duration was admitted on $\mathrm{Na}$ - tional Cardiovascular Center on September 17, 1981 because of the loss of consciousness. The patient underwent a clipping of aneurysma in the right middle cerebral artery and anterior communicating artery on August 28, 1978 in this hospital. Since the operation, $100 \mathrm{mg}$ phenytoin and $200 \mathrm{mg}$ mephobarbital twice daily had been administered, and he kept daily activity without any neural symptoms. His diabetes had been treated on diet since the onset, but his control was not satisfactory. $50 \mathrm{~g}$ oral glucose tolerance test performed in June, 1978 showed 199, 301, 401, 453, 413 and $363 \mathrm{mg} / \mathrm{dl}$ of plasma glucose, before, 30, 60, 90,120 and 180 minutes after loading, respectively. The fasting plasma glucose in March, 1979 and February, 1981 were 162 and $228 \mathrm{mg} / \mathrm{dl}$, respectively. Three days before admission he complained of increasing dysphagia for solid foods, and two days before he could hardly swallow saliva. On admission, diagnosis of non-ketotic diabetic coma was made. The brain CT scanning showed no changes compared with that of the previous admission. His weight was $63 \mathrm{~kg}, 4 \mathrm{~kg}$ of weight loss since the beginning of dysphagia, and height was $170 \mathrm{~cm}$. His blood pressure was $120 / 80 \mathrm{mmHg}$ and showed sinus tachycardia. His plasma glucose was $864 \mathrm{mg} / \mathrm{dl}$ while no ketones in urine, and his plasma electrolytes were

Table 1. Laboratory findings on admission

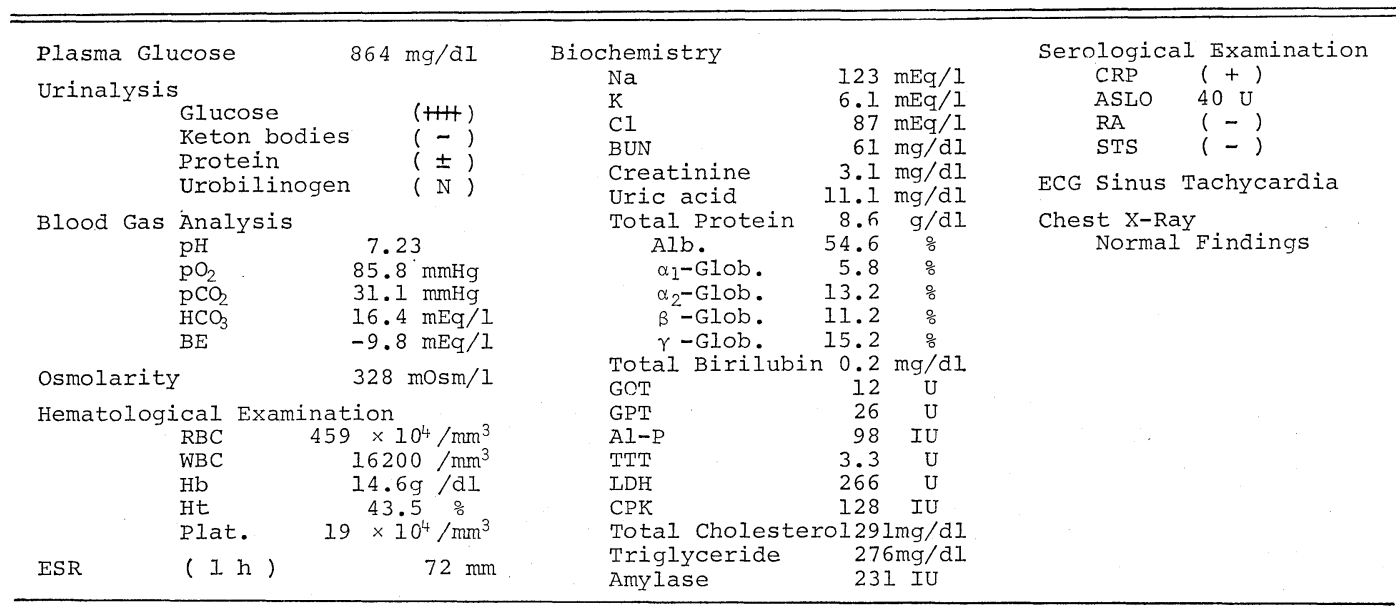


sodium $123 \mathrm{mEq} / 1$, potassium $6.1 \mathrm{mEq} / 1$, chloride $87 \mathrm{mEq} / 1$ and his blood urea nitrogen was $61 \mathrm{mg} / \mathrm{dl}$ with the slight increase of creatinine. Hematological examination showed leucocytosis but heamatocrit value was not so high because of the previous anemia. The osmolarity was $328 \mathrm{mOsm} / 1$ (Table 1). Intravenous fluid and continous insulin infusion resulted in a recovery of diabetic coma, and a normalization of plasma glucose, blood urea nitrogen and electrolytes were observed. In the course, intubation and tracheotomy was necessary to keep the air way patient preventing a further development of swallowing pneumonia. At 12 hospital day, dysphagia began to subside gradually and on October 3 his dysphagia disappeared completely (Fig. 1). Normal barium swallowing was noted radiologically after his recovery. Ophtalmo- logical examination revealed the mild diabetic changes (Scott Ia) and renal function was normal. Vibratory sensation was decreased slightly, while almost normal tendon reflexes were observed. He had never had a habit of smoking and taking alcohol.

His diabetes was easily controled with diet and exercise, and glucosuria was not observed.

In order to confirm the presence and assess the severity of the autonomic nerve dysfunction, we tried simple, non-invasive and reproducible new generation tests based on cardiovascular reflexes which are assumed to reflect autonomic nerve changes in other systems ${ }^{13}$. The results of the tests are shown in Table 2 and Figure 2. Postural fall of blood pressure was noted but it was less than $30 \mathrm{mmHg}$ in systolic blood pressure $^{10)}$. On the other hand, diastolic pres-

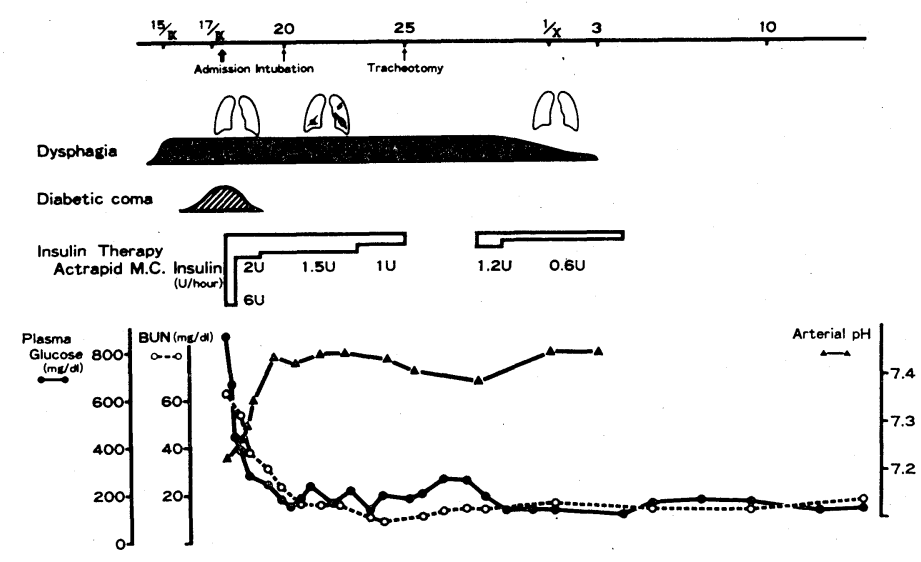

Fig. 1. Glinical course of diabetic coma with dysphagia.

Table 2. Autonomic nerve function after the recovery from diabetic coma

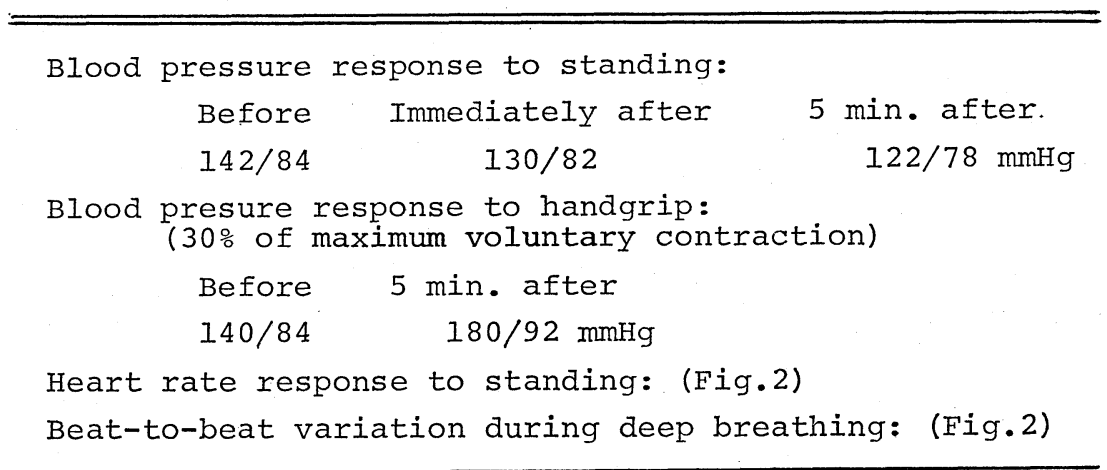



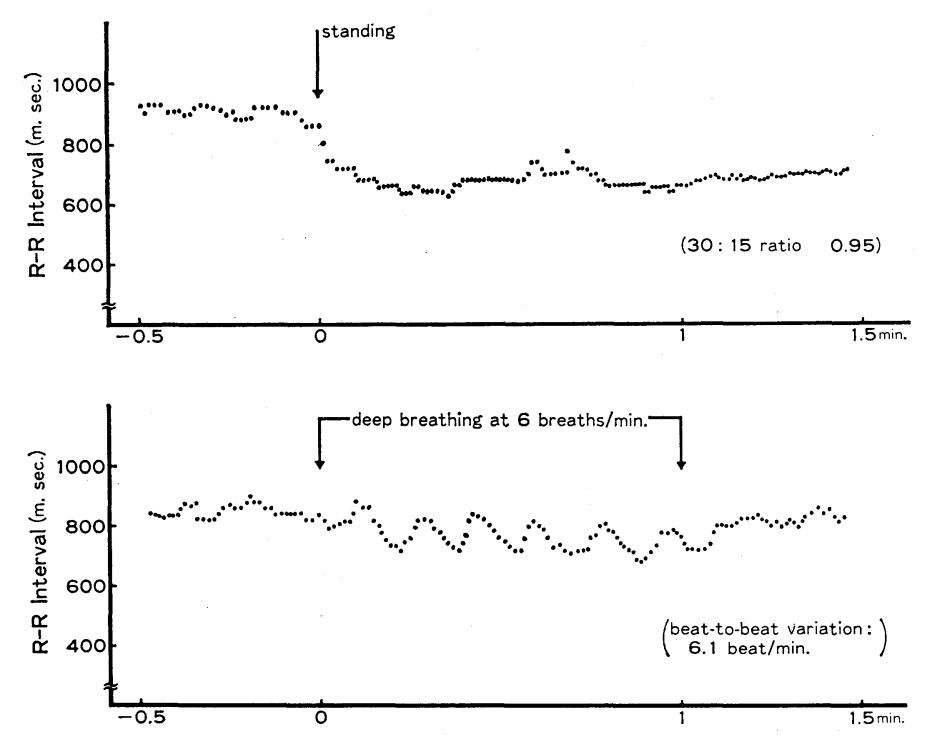

Fig. 2. Heart rate response to standing, and beat-to-beat variation during deep breathing.

sure rose only by $8 \mathrm{mmHg}$ in response to handgrip, and was defined as abnormal ${ }^{14)}$. Both heart rate response to standing and beat-to-beat variation during deep breathing were shown as abnormal. "30:15" ratio calculated from the ratio of the lengths of the R-R intervals at beat 30 and 15 after standing indicate 0.95 , and satisfied the criteria of autonomic neuropathy of the values of 1.00 or 1 ess $^{11)}$. Beat-to-beat variation during 6 successive cyclic breaths was 6.1 beats/min., which regarded as abnormal, since normal subjects showes more than 10 beats $/ \min .{ }^{12)}$.

\section{DISCUSSION}

Esophageal motility dysfuction in diabetes is well known but symptoms such as dysphagia, retrosternal discomfort and heartburn are uncommon ${ }^{1-7)}$. Diminished pharyngeal and esophageal contractions and reduced lower sphincter pressure are demonstrated in diabetics by manometic and radiological studies. These motility changes are similar to those of animals with vagal section $^{8)}$, and from the pharmacological studies using cholinergic drugs, lesions in preganglionic fiber of the vagus has been indicated to be predominant in diabetics ${ }^{6)}$. To our knowledge, no case of the association of diabetic coma with severe dysphagia was documented. Recently Burke reported ${ }^{95}$ two cases of hyperglycemic hyperosmolar non-ketotic diabetic coma preceded by severe dysphagia. Both patients were elderly female type II diabetics with duration of 10 years, and they had been administered diuretics. The present male case with nonketotic diabetic coma was also preceded by severe dysphagia, and showed the same type of diabetes, clinical duration and age, but he had never received diuretic treatment (Table 3). In Burke's cases, the relationship between the severity of autonomic nerve dysfunction and dysphagia has not been studied. It has been recognized that sympathetic damage can be demonstrated by a postural fall of blood pressure ${ }^{10)}$ or an abnormal response to handgrip ${ }^{10)}$, whereas, parasympathetic damage is shown by abnormal vagal function such as abnormal heart rate response to standing ${ }^{11)}$ or a decrease of beat-to-beat variation in heart rate during deep breathing ${ }^{15-16)}$. Therefore, the abnormality of parasympathetic nerve rather than the sympathetic nerve seemed 
Table 3. Comparison of the previously reported two cases (case 1, case 2) and our case (case 3) with dysphagia associated with diabetic coma

\begin{tabular}{l|c|c|c}
\hline & Case I & Case 2 & Case 3 \\
\hline Age & 58 & 70 & 62 \\
Sex & Female & Female & Male \\
Duration of diabetes (years) & $I 1$ & 14 & II \\
Types of diabetes & Type II & Type II & Type II \\
Dysphagia on admission & $(+)$ & $(+)$ & $(+)$ \\
Retonuria & $(-)$ & $(-)$ & $(-)$ \\
Plasma glucose (mg/dl) & 1152 & 1017 & 864 \\
Osmotic pressure (mOsm/1) & 385 & 357 & 328 \\
Serum Na (mEq/I) & 108 & 139 & 123 \\
Bicarbonate (mEq/1) & 22 & 27 & 16 \\
Diuretics before admission & $(+)$ & $(+)$ & $(-)$ \\
\hline
\end{tabular}

to be predominant in our case. But these abnormalities was not so severe compared with the late stage autonomic neuropathy in diabetes. More severe autonomic nerve dysfunction might have been present at the onset of dysphagia because these tests were carried out after the recovery. Considering no history to taking diuretics in the present case, dysphagia might be induced by autonomic nerve dysfunction which was derived from the disturbed diabetic control. Furthermore, it is suggested that dysphagia might have accelerated the development of the disturbed diabetic control to coma through the poor oral intake. Dysphagia might also be caused rather commonly in diabetics with previous evidence of cerebrovascular accident or operation even if they have no apparant cranial nerve symptoms.

These observations together with Burke's one indicate that progressive dysphagia in elderly diabetics, with or without taking diuretics or previous evidence of cerebrovascular accident, should be treated carefully albeit the severity of diabetes or diabetic neuropathy to prevent the development to diabetic coma.

\section{REFERENCES}

1) Cobum WM: Oesophageal motility in diabetes. Lancet II : 261, 1967.

2) Mandelstam P, Lieber A: Esophageal dysfunction in diabetic neuropathy-gastrentero- phthy. J.A. M. A. $201:$ 582, 1967.

3) Mandelstam P, Siegel CI, Lieber A, et al: The swallowing disorder in patients with diabetic neuropathy-gastroenteropathy. Gastroenterology 5'bे : 1, 1969.

4) Silber W: Diabetes and oesophageal dysfunction. Brit Med J III : 688, 1969.

5) Vela AR, Balart LA : Esophageal motor manifestations in diabetes mellitus. Am J Surg 119: 21, 1970.

6) Stewart IM, Hosking DJ, Preston BJ, et al : Oesophageal motor changes in diabetes mellitus. Thorax 31: 278, 1976.

7) Hollis JB, Castell DO, Braddom RL: Esophageal function in diabetes mellitus and its relation to peripheral neuropathy. Gastroenterology 73 : 1098, 1977.

8) Goyal RK, Spiro HM: Esophageal function in diabetes mellitus. Ann Intern Med 72: 281, 1970.

9) Burke BJ : Hyperglycemic hyperosmolar nonketotic diabetic coma presenting as severe dysphagia. Brit Med J 14: 1421, 1980.

10) Ewing DJ, Campbell IW, Burt AA, et al: Vascular reflexes in diabetic autonomic neuropathy. Lancet II : 1354, 1973.

11) Ewing DJ, Campbell IW, Murray A, et al: Immediate heart rate response to standing: simple test for autonomic neuropathy in diabetics. Brit Med J I: 145, 1978.

12) Page MM, Watkins PJ: The heart in diabetes: autonomic neuropathy and cardiomyopathy. Glin Endocrinol Metab 6: 377, 1977.

13) Ewing WJ: Cardiovascular reflexes and autonomic neuropathy. Clin Sci Mol Med $55: 321,1978$.

14) Ewing DJ, Irving JB, Kerr F, et al : Car- 
diovascular responses to sustained handgrip in normal subjects and patients with diabetes with diabetes mellitus: a test of autonomic function. Clin Sci Mol Med 46: 295, 1974.

15) Wheeler $T$, Watkins PJ: Cardiac denervation in diabetes. Brit Med J IV : 584, 1973.
16) Bennett $T$, Farquhar IK, Hosking DJ, et al: Assessment of methods for estimating autonomic nervous control of heart in patients with diabetes mellitus. Diabetes 27: 1167, 1978. 